\title{
REGADÍO Y SERVICIOS ECOSISTÉMICOS. UNA VALORACIÓN INTEGRAL
}

\author{
Francisco Alcon $^{a^{*}}$, José A. Zabala ${ }^{a}$, Víctor Martínez-García ${ }^{a}$, José A. Albaladejo-Garcíab, Erasmo I. \\ López-Becerra $^{\mathrm{a}}$, María D. de-Miguela ${ }^{\mathrm{a}}$, José M. Martínez-Paz
}

a Departamento de Economía de la Empresa. Universidad Politécnica de Cartagena. (Cartagena, joseangel.zabala@upct.es, victor.martinez@upct.es, erasmo.lopez@upct.es, ․md.miguel@upct.es, francisco.alcon@upct.es)

${ }^{b}$ Departamento de Economía Aplicada. Universidad de Murcia. (Murcia, joseantonio.albaladejo@um.es, jmpaz@um.es)

\section{Resumen}

El aporte de agua de riego condiciona la provisión de servicios y contraservicios ecosistémicos de los sistemas agrarios y, en consecuencia, la contribución de esta actividad al bienestar humano. El objetivo del trabajo es valorar económicamente la provisión de servicios y contraservicios ecosistémicos asociados al agua de riego en el área mediterránea, a partir del estudio y evaluación de sistemas de cultivos de secano y de regadío, diferenciando a su vez entre regadíos tradicionales y modernizados. Como caso de estudio se utiliza el cultivo del limonero en la Cuenca del Segura (sudeste España) considerando el regadío tradicional del Valle de Ricote y el regadío modernizado del Campo de Cartagena, y fijando el almendro como la alternativa en secano al cultivo de este cítrico. Para cada cultivo y zona se evalúan una serie de indicadores biofísicos, asociados a la provisión de los distintos servicios y contraservicios, que son a su vez valorados económicamente a través de métodos de mercado y de no-mercado.

Los resultados preliminares muestran que los valores de mercado en el regadío modernizado son superiores, aunque los valores de no-mercado del regadío tradicional pueden llegar a compensar estas diferencias. Así, la consideración del valor integral de los servicios ecosistémicos del agua de riego puede contribuir a mejorar la gestión de este recurso en aras a maximizar su contribución al bienestar social.

Palabras clave: Bienestar humano, Recursos hídricos, Servicios ecosistémicos, Valoración de no-mercado.

\section{Introducción y objetivos}

El regadío es el principal usuario del agua dulce, consumiendo el $85 \%$ del total de agua en todo el mundo [WWAP (2016)]. Sin embargo, la menor disponibilidad de recursos hídricos debido al cambio climático, junto con la creciente demanda de alimentos de la población, requerirá de una adaptación de los sistemas agrarios - agroecosistemas de regadío que traiga consigo el aumento de la productividad y la mejora de la gestión de ese recurso. Por otro lado, los agroecosistemas proporcionan a la sociedad más que alimentos y contribuyen positiva y negativamente al bienestar humano a través de los servicios (SE) y contraservicios ecosistémicos (CSE) que proveen. La presión antrópica ejercida por las prácticas de cultivo impacta de lleno en las funciones y capacidades de los agroecosistemas, lo que se traslada finalmente en los flujos de SE y CSE a la sociedad. En este sentido, el aporte de agua de riego constituye no solo el sustento de la agricultura de regadío, sino también un factor condicionante y determinante en la provisión de SE y CSE de la agricultura.

El enfoque de los servicios ecosistémicos proporciona, por tanto, un nuevo marco para la valoración y la evaluación del agua en la agricultura. Integrar el valor de los SE y CSE proveídos por los agroecosistemas en las decisiones públicas permite incorporar los beneficios y costes derivados del uso de agua en la agricultura. Así, la gestión y asignación del agua es abordada no sólo desde un punto de vista económico, sino también ambiental y social, perspectiva que es de gran relevancia en zonas con escasez de agua, como es el caso de la región mediterránea.

Dado este contexto, el objetivo del presente trabajo es valorar económicamente la contribución del agua de riego a la provisión de SE y CSE de los agroecosistemas, y, con ello su contribución al bienestar, en la agricultura mediterránea. Para ello, se ha valorado la provisión de SE y CSE de agroecosistemas de regadío y secano, distinguiendo, a su vez, entre regadío tradicional y modernizado, evaluando el impacto de distintos modelos en la gestión del agua de riego en la valoración. Como caso de estudio, se ha tomado de referencia la Región de Murcia (España), dentro de la Cuenca del Segura, una zona caracterizada por tener el índice de explotación hídrico más alto de Europa. Dentro de la misma se han seleccionado dos subsistemas representativos de los tipos de gestión de los recursos hídricos: el regadío tradicional, característico del Valle de Ricote (VR), y el regadío modernizado, localizado en el Campo de Cartagena (CC). Como cultivos de referencia se ha tomado el limonero en el caso del regadío -cultivo leñoso más extendido y común para ambos subsistemas-, y el almendro, en un régimen de secano, entendiendo este último como el cultivo leñoso sustitutivo más frecuente en caso de ausencia de riego. 


\section{Metodología}

La metodología empleada para valorar la contribución del agua de riego al bienestar humano parte de la valoración de los SE y CSE proporcionados por los agroecosistemas de regadío y secano para el caso de estudio. Para ello, se combinó la cuantificación de indicadores biofísicos con técnicas de valoración de mercado y no-mercado para cada uno de los SE y CSE a valorar. La selección de los SE y CSE siguió la propuesta de Zabala et al. (2021a), donde se lleva a cabo una readaptación de los principales marcos de trabajo para los servicios ecosistémicos al caso particular de los agroecosistemas, con especial atención a los localizados en las regiones mediterráneas. Los indicadores biofísicos son empleados como proxy cuantitativo de los flujos de servicios y contraservicios proporcionados por los agroecosistemas analizados. El Cuadro 1 presenta los SE y CSE analizados, así como los indicadores biofísicos y el método de valoración seguido para su cuantificación económica. Se ha establecido 2019 como año de referencia.

Cuadro 1. Servicios y contraservicios analizados, indicadores biofísicos y métodos de valoración

\begin{tabular}{|c|c|c|c|c|}
\hline & SE/CSE & Indicador biofísico & Unidades & Método de valoración [Fuente] \\
\hline \multirow[t]{2}{*}{ 警 } & Alimentos (SE) & $\begin{array}{l}\text { Factores de producción consumidos por } \\
\text { la actividad agrícola (fertilizantes, } \\
\text { pesticidas, maquinaria, etc.) }\end{array}$ & Unidades/ha & $\begin{array}{l}\text { Experimentos de elección } \\
\text { 「Zabala et al. (2021b)] } \\
\text { Precios de mercado (margen } \\
\text { neto, incluyendo costes de } \\
\text { oportunidad) } \\
\text { [Fernández et al. (2020)] }\end{array}$ \\
\hline & Agua de riego (CSE) & $\begin{array}{l}\text { Consumo de agua de riego por parte de } \\
\text { los cultivos }\end{array}$ & $\mathrm{m}^{3} / \mathrm{ha} / \mathrm{año}$ & $\begin{array}{l}\text { Experimentos de elección } \\
{[\text { Zabala et al. }(2021 \mathrm{~b})]}\end{array}$ \\
\hline \multirow{5}{*}{ 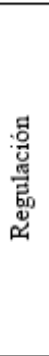 } & $\begin{array}{l}\text { Emisión de contaminantes a la } \\
\text { atmósfera (CSE) } \\
\text { Regulación del clima global (SE) }\end{array}$ & $\begin{array}{l}\text { Balance de carbono. Diferencia entre la } \\
\text { emisión y secuestro de gases de efecto } \\
\text { invernadero }\end{array}$ & $\mathrm{tCO}_{2 \mathrm{eq}} \mathrm{ha} / \mathrm{año}$ & $\begin{array}{l}\text { Transferencia de beneficios } \\
\text { [Resvesz et al. (2017)] }\end{array}$ \\
\hline & Regulación del clima local (SE) & $\begin{array}{l}\text { Reducción de temperatura en la } \\
\text { superficie debido a la actividad agrícola }\end{array}$ & ${ }^{\circ} \mathrm{C}$ & $\begin{array}{l}\text { Experimentos de elección } \\
\text { [Zabala et al. }(2021 \mathrm{~b})]\end{array}$ \\
\hline & $\begin{array}{l}\text { Purificación del agua y } \\
\text { tratamiento de residuos (CSE) }\end{array}$ & $\begin{array}{l}\text { Concentración de nitratos en los } \\
\text { acuiferos }\end{array}$ & $\mathrm{mg} \mathrm{NO}_{3}-1$ & $\begin{array}{l}\text { Experimentos de elección } \\
\text { [Zabala et al. }(2021 \mathrm{~b})]\end{array}$ \\
\hline & Mantenimiento del suelo (CSE) & $\begin{array}{l}\text { Pérdida del suelo fértil debido al viento } \\
\text { y precipitación (erosión) }\end{array}$ & t/ha/año & $\begin{array}{l}\text { Transferencia de beneficios } \\
\text { [Rodríguez-Entrena et al. (2014)] }\end{array}$ \\
\hline & Biodiversidad (SE/CSE) & $\begin{array}{l}\text { Riqueza de aves respecto del potencial } \\
\text { del agroecosistema (Indice de Margalef) }\end{array}$ & $\%$ & $\begin{array}{l}\text { Experimentos de elección } \\
\text { [Zabala et al. (2021b)] }\end{array}$ \\
\hline \multirow{4}{*}{ 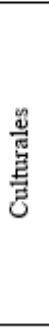 } & Cultura, arte y diseño (SE) & $\begin{array}{l}\text { Presencia de elementos culturales } \\
\text { asociados a la agricultura }\end{array}$ & - & $\begin{array}{l}\text { Transferencia de beneficios } \\
\text { [Garcia-Llorente et al. }(2012)]\end{array}$ \\
\hline & Valores estéticos (SE) & $\begin{array}{l}\text { Presencia de otros cultivos leñosos } \\
\text { distintos del principal (heterogeneidad } \\
\text { del paisaje) }\end{array}$ & $\%$ & $\begin{array}{l}\text { Transferencia de beneficios } \\
\text { [Arriaza et al. (2008)] }\end{array}$ \\
\hline & $\begin{array}{l}\text { Oportunidades para ocio y } \\
\text { turismo (SE) }\end{array}$ & $\begin{array}{l}\text { Oportunidad para el disfrute de } \\
\text { actividades de ocio en el entorno del } \\
\text { agroecosistema }\end{array}$ & - & $\begin{array}{l}\text { Experimentos de elección } \\
\text { [Zabala et al. }(2021 \mathrm{~b})]\end{array}$ \\
\hline & Desarrollo cognitivo (SE) & $\begin{array}{l}\text { Creación de puestos de trabajo por la } \\
\text { actividad agrícola }\end{array}$ & UTA/ha/año & $\begin{array}{l}\text { Precios sombra } \\
\text { [European Commission (2014)] }\end{array}$ \\
\hline
\end{tabular}

Fuente: Elaboración propia

Los valores para los indicadores biofísicos se han estimado a partir de datos geofísicos (ej. erosión, riqueza de aves, heterogeneidad del paisaje), obtenidos directamente de mediciones en campo o de entrevistas con agricultores y/o técnicos agrarios.

El valor económico total de cada subsistema se ha calculado a partir de los valores de mercado y nomercado para cada uno de los SE y CSE considerados, tal como recoge la expresión siguiente:

$$
V E T_{i}=\sum V E_{n}=\sum V E_{\text {mercado }}+\sum V E_{n o-\text { mercado }}
$$

Donde $V E T_{i}$ representa el valor económico total del subsistema $i$-expresado en $€ /$ ha-, y $V E_{n}$ es el valor económico que proporciona cada uno de los $n$ SE y CSE considerados. El valor de dichos SE y CSE se ha calculado con el fin de recojan el impacto en el bienestar humano de cada uno de los subsistemas considerados. Señalar como el SE de provisión de alimentos contribuye no solo a la seguridad alimentaria de la población (valor de no-mercado) sino también a la renta de los agricultores (valor de mercado), de ahí que su valor se ha obtenidos agregando el resultante de dos métodos.

Así, la contribución del agua de riego al bienestar puede expresarse a través de la diferencia en el valor económico de los subsistemas de secano y regadío:

$$
\Delta V E T_{\text {agua }}=\sum V E_{\text {regadío }}-\sum V E_{\text {secano }}
$$

\section{Resultados}


El Cuadro 2 recoge, de forma sintética, los principales resultados obtenidos. En cuanto al agroecosistema de secano, independientemente del subsistema considerado, las principales contribuciones a su VET provienen del valor de no-mercado de los SE y CSE que proporciona. El valor de mercado para el subsistema tradcional de secano llega a ser negativo al considerar todos los costes a los que incurren los agricultores, parte de ellos derivados del coste de oportunidad de la mano de obra no asalariada. El valor de los SE y CSE de regulación supone entre el $50 \%$ y el $75 \%$ del VET de los subsistemas de secano, mientras que los SE culturales rondan el $20 \%$. Ello contrasta con los resultados obtenidos para el agroecosistema de regadío, donde los SE y CSE de provisión, y en particular los SE de mercado, representan el grueso de la contribución de cada subsistema al bienestar humano y social, cuya importancia ronda el 70 $\%$ y $80 \%$ los subsistemas tradicional y modernizado, respectivamente. En este sentido, los SE y CSE de regulación y culturales tienen una importancia similar en cuanto al valor de cada subsistema de regadío, que se sitúa sobre el $15 \%$ y el $10 \%$ para VR y CC, respectivamente. De este modo, se deja constancia que la contribución de la agricultura de regadío a la sociedad reside principalmente en su capacidad para proveer alimentos, mientras que la agricultura de secano debe su transcendencia a la regulación de los procesos ecológicos que se suceden en el agroecosistema.

Cuadro 2. Indicadores biofísicos, valor económico (VET) y contribución del agua de riego (DVETagua)

\begin{tabular}{|c|c|c|c|c|c|c|c|}
\hline & & & & Secano ( & lmendro) & Regadío ( & limonero) \\
\hline & SE/CSE & Indicador & $\begin{array}{l}\text { Unidades } \\
(\sim / \text { ha/año })\end{array}$ & $\begin{array}{l}\text { Trad. } \\
\text { (VR) }\end{array}$ & $\begin{array}{l}\text { Modern. } \\
\text { (CC) }\end{array}$ & $\begin{array}{l}\text { Trad. } \\
\text { (VR) }\end{array}$ & $\begin{array}{l}\text { Modern. } \\
\text { (CC) }\end{array}$ \\
\hline & & Producción & $\mathrm{Kg}$ & 239,58 & 94,04 & $34.600,00$ & $49.120,00$ \\
\hline 苟 & Alimentos (SE) & Valor no-mercado & $€$ & 237,11 & 97,47 & 635,73 & 653,84 \\
\hline 3 & & Valor mercado & $€$ & 285,17 & $-45,29$ & $7.096,05$ & $10.791,98$ \\
\hline 品 & & Consumo de agua & $\mathrm{m}^{3}$ & & & $4.909,40$ & $6.234,60$ \\
\hline & Agua de riego (CSE) & Valor no-mercado & $€$ & & & 82,35 & $-238,60$ \\
\hline & $\begin{array}{l}\text { Emisión de contaminantes a la } \\
\text { atmósfera (CSE) }\end{array}$ & Balance de carbono & $\mathrm{t} \mathrm{CO}_{2 \mathrm{eq}}$ & 16,13 & 15,26 & 30,54 & 25,07 \\
\hline & Regulación del clima global (SE) & Valor no-mercado & $€$ & 564,35 & 518,30 & $1.093,25$ & $1.025,49$ \\
\hline & Regulación del clima local (SE) & $\begin{array}{l}\text { Reducción } \\
\text { temperatura }\end{array}$ & ${ }^{\circ} \mathrm{C}$ & & & $-0,55$ & $-1,20$ \\
\hline 吾 & & Valor no-mercado & $€$ & & & 9,40 & 20,54 \\
\hline 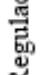 & Purificación del agua y & $\begin{array}{l}\text { Contaminación de } \\
\text { acuiferos }\end{array}$ & $\mathrm{mg} \mathrm{NO}{ }_{3}^{-} / 1$ & 1,45 & 33,38 & 9,63 & 104,30 \\
\hline & tratamiento de residuos (CSE) & Valor no-mercado & $€$ & $-0,65$ & $-17,15$ & $-6,36$ & $-96,64$ \\
\hline & & Erosión & $\mathrm{t}$ suelo & 69,31 & 18,57 & 46,16 & 5,47 \\
\hline & Mantenimiento del suelo (CSE) & Valor no-mercado & $€$ & $-75,70$ & $-20,28$ & $-50,41$ & $-5,97$ \\
\hline & & Riqueza aves & $\%$ & 100,00 & 83,70 & 100,00 & 73,82 \\
\hline & Biodiversidad (SE/CSE) & Valor no-mercado & $€$ & 353,37 & 295,75 & 361,20 & 314,61 \\
\hline & Cultura, arte y diseño (SE) & Valor no-mercado & $€$ & 51,26 & 51,26 & 92,65 & 11,74 \\
\hline$\stackrel{\mathscr{H}}{=}$ & Valores estéticos (SE) & $\begin{array}{l}\text { Heterogeneidad } \\
\text { paisaje }\end{array}$ & $\%$ & 13,16 & 1,60 & 23,00 & 14,72 \\
\hline 莺 & & Valor no-mercado & $€$ & 29,08 & 3,54 & 50,82 & 32,53 \\
\hline 壱 & $\begin{array}{l}\text { Oportunidades para ocio y } \\
\text { turismo (SE) }\end{array}$ & Valor no-mercado & $€$ & 100,14 & 75,21 & 97,17 & 82,14 \\
\hline & Desarrollo cognitivo (SE) & Puestos de trabajo & UTA & 0,02 & 0,02 & 0,33 & 0,28 \\
\hline & & Valor no-mercado & $€$ & $\frac{100,70}{164484}$ & $\frac{80,06}{103887}$ & $\frac{1.436,35}{10.80810}$ & $\frac{1.203,95}{1270561}$ \\
\hline & & $\begin{array}{l}\text { VET } \\
\triangle \text { VETagua }\end{array}$ & $\begin{array}{l}€ \\
€\end{array}$ & $1.644,84$ & $1.038,87$ & $\begin{array}{l}10.898,19 \\
9.253,35\end{array}$ & $\begin{array}{l}13.795,61 \\
12.756,74\end{array}$ \\
\hline
\end{tabular}

Fuente: Elaboración propia

La mayor contribución del agua de riego al valor de la provisión de SE y CSE, en términos absolutos, se da en el subsistema de regadío modernizado. Así, transformar agricultura de leñosos en secano a regadío genera un impacto en el bienestar superior a $12.500 € /$ ha y año en el CC, y a $9.000 € /$ ha y año en el VR. Tales diferencias se ven reducidas cuando el análisis se realiza de un modo relativo, trabajando en términos de productividad económica del agua. De hecho, ambos subsistemas revelan productividades del agua similares de en torno a $2,22 € / \mathrm{m}^{3}$. Sin embargo, el aporte de agua de riego al subsistema modernizado implica una mayor productividad de mercado del agua que en el regadío tradicional, al contrario de lo que sucede con la productividad de no mercado del agua.

\section{Conclusiones}

Este trabajo ha permitido valorar los principales SE y CSE que los agroecosistemas proporcionan a la sociedad, siendo el agua de riego el eje central del análisis. Los resultados ponen de manifiesto la contribución del agua de riego al valor de los SE y CSE de los agroecosistemas, y cómo las prácticas 
culturales, ligadas a la gestión de los recursos hídricos por los agricultores en un subsistema tradicional o modernizado, establecen importantes diferencias en los beneficios que genera.

Los resultados obtenidos, más allá de valores monetarios concretos, generan un nuevo marco de evaluación de políticas de gestión de los recursos hídricos, especialmente útiles en zonas de escasez hídrica como la Región de Murcia, ya que tienen en cuenta las distintas fuentes de bienestar que los agroecosistemas generan.

\section{Agradecimientos}

Este trabajo se ha realizado en el marco del proyecto 20912/PI/18 financiado por la "Fundación SénecaAgencia de Ciencia y Tecnología de la Región de Murcia". José A. Zabala, Víctor Martínez-García y José A. Albaladejo-García agradecen la ayuda recibida del MEFP (FPU16/03473 | FPU19/05143 | FPU 16/03562).

\section{Bibliografía}

Arriaza, M., Gómez-Limón, J.A., Kallas, Z. y Nekhay, O. (2008). "Demand for non-commodity outputs from mountain olive groves". Agricultural Economics Review, 9(1): 5-23.

European Commission. (2014). Guide to cost-benefit analysis of investment projects. Publications Office of the European Union, Luxembourg.

Fernández, J.E., Alcon, F., Diaz-Espejo, A., Hernandez-Santana, V. y Cuevas, M.V. (2020). "Water use indicators and economic analysis for on-farm irrigation decision: A case study of a super high density olive three orchard". Agricultural Water Management, 237: 106074.

García-Llorente, M., Martín-López, B., Iniesta-Arandia, I., López-Santiago, C.A., Aguilera, P.A. y Montes, C. (2012). "The role of multi-functionality in social preferences toward semi-arid rural landscapes: An ecosystem service approach". Environmental Science \& Policy, 19-20: 136-146.

Revesz, R., Greenstone, M., Hanemann, M., Livermore, M., Sterner, T., Grab, D., Howard, P. y Schwartz, J. (2017). "Best cost estimate of greenhouse gases". Science, 357(6352): 655.

Rodríguez-Entrena, M., Espinosa-Goded, M. y Barreiro-Hurlé, J. (2014). "The role of ancillary benefits on the value of agricultural soils carbon sequestration programmes: Evidence from a latent class approach to Andalusian olive groves". Ecological Economics, 99: 63-73.

WWAP (Programa Mundial de Evaluación de los Recursos Hídricos de las Naciones Unidas). (2016). Informe de las Naciones Unidas sobre el Desarrollo de los Recursos Hídricos en el Mundo 2016: Agua y Empleo. UNESCO CLD, París.

Zabala, J.A., Martínez-Paz, J.M. y Alcon, F. (2021a). “A comprehensive approach for agroecosystem services and disservices valuation". Science of The Total Environment, 768: 144859.

Zabala, J.A., Martínez-Paz, J.M. y Alcon, F. (2021b). "Integrated valuation of semiarid Mediterranean agroecosystem services and disservices”. Ecological Economics 184: 107008. 